\title{
The Comparison of Speech Intelligibility between the Cochlear Implanted and Normal-Hearing Children
}

\author{
Asma Torfi ${ }^{1}$, Fatemeh Jahangirimehr ${ }^{2}$, Hossein Bagheripour ${ }^{1}$, Arash Bayat $^{1,3}$, Nader Saki ${ }^{1} * \mathbb{D}$ \\ Received: 23 Jan 2021 \\ Published: 30 Oct 2021
}

Abstract

Background: Following the development of new hearing technologies, assessment of speech intelligibility in hearing-impaired children is of great interest. The main purpose of this study was to compare speech intelligibility and auditory perception abilities in children with normal-hearing $(\mathrm{NH})$ and children with hearing aid (HA) and cochlear implant (CI).

Methods: This analytic cross-sectional study consisted of 60 Persian-speaking children aged 5 to 7-years. Participants were

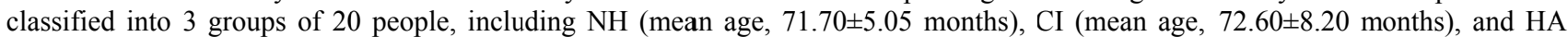
(mean age, $71.45 \pm 10.56$ months) children. The speech intelligibility rating (SIR) and categories of auditory performance (CAP) tests were conducted for all children to measure their speech intelligibility and auditory perception, respectively. A one-way analysis of variance (ANOVA) test was used to compare CAP and SIR scores among the 3 groups.

Results: The mean SIR score in the NH children was significantly higher than the HA $(p=0.002)$ and CI $(p=0.009)$ groups. However, these differences between the HA and CI children were not significant ( $\mathrm{p}=0.885)$. We found a significant difference between the CAP scores in the 3 groups $(\mathrm{p}=0.038)$. Furthermore, the post hoc analysis results indicated that the mean CAP scores in NH children were significantly higher than the HA and CI participants. Based on the results, the speech intelligibility and auditory performance abilities in $\mathrm{NH}$ children were significantly greater than the hearing-impaired (CI and HA) children. However, these abilities between HA and CI users were not significantly different.

Conclusion: Based on the results, the early acoustical amplification on auditory and speech functions in children with hearing loss is of paramount importance.

Keywords: Speech Intelligibility, Hearing Aids, Cochlear Implant, Auditory Performance

Conflicts of Interest: None declared

Funding: This study was supported by a grant from the Deputy of Research, AJUMS, Ahvaz, Iran (Grant Number: HRC-9807).

*This work has been published under CC BY-NC-SA 1.0 license.

Copyright $($ Iran University of Medical Sciences

Cite this article as: Torfi A, Jahangirimehr F, Bagheripour H, Bayat A, Saki N. The Comparison of Speech Intelligibility between the Cochlear Implanted and Normal-Hearing Children. Med J Islam Repub Iran. 2021 (30 Oct);35:143. https://doi.org/10.47176/mjiri.35.143

\section{Introduction}

Several studies have shown that good speech intelligibility, or how well others can recognize one's speech, is an

Corresponding author: Dr Nader Saki, ahvaz.ent@gmail.com

1. Hearing Research Center, Clinical Sciences Research Institute, Ahvaz Jundishapur of Medical Sciences, Ahvaz, Iran

2. Public Health School, Ahvaz Jundishapur University of Medical Sciences, Ahvaz, Iran

3. Musculoskeletal Rehabilitation Research Center, Ahvaz Jundishapur University of Medical Sciences, Ahvaz, Iran important speech-language outcome for individuals with hearing impairment. Good speech intelligibility ability

$\uparrow$ What is "already known” in this topic:

- Detailed and quantitative scrutiny of hearing-impaired children is necessary for designing their training programs, and there is a controversy about speech intelligibility of the children with cochlear implant and hearing aids.

- There are not enough studies in Persian language on speech intelligibility in hearing impaired children.

$\rightarrow$ What this article adds:

- A great communication benefits are achieved by early implantation. The results exemplify the importance of enhanced social environments provided by cochlear implants.

- Children who had received CIs exceed achievement levels obtained by children who use hearing aids with respect to the comprehension and production of sentences. 
also entails mastery of multiple foundational spoken language skills, such as speech comprehension, phonological awareness, and intonation, and it is positively associated with a range of cognitive skills (eg, visual attention) and psychosocial behaviours (eg, emotional adjustment) $(1,2)$. Various factors can influence speech intelligibility, including the level of communication skill (single word or continuous speech), the listener's familiarity with the speaker's speech, and communication cues for the listener (familiar text or unfamiliar text) (3-6).

Hearing loss is an important factor that may negatively affect children's speech intelligibility. Hearing-impaired children may not be able to distinguish between the different phonemes, and because of the lack of auditory feedback, their speech production may be confusing $(7,8)$. In recent years, technological advances have led to the development of intelligent hearing aids (HAs) and multichannel cochlear implants (CIs) that permit children who are profoundly hearing-impaired access to environmental sounds and information about spoken language and improve their speech intelligibility skills.

The cochlear implant is a safe and standard surgical procedure for patients with severe-to-profound sensorineural hearing loss (SNHL) who received a limited benefit from their hearing aids. It could help to restore auditory perception and cognition via electrical stimulation of the auditory nerve fibers $(9,10)$. It has been indicated that CIs can improve speech intelligibility in hard of hearing children. Most and Peled (11) investigated understanding speech prosodic features between a group of cochlear implanted children and 2 groups of hearing aid users with severe and profound SNHL. Their findings demonstrated that children who were fitted with hearing aids showed a better performance in understanding the prosodic characteristics compared with the implanted children. In another study, Rezaei et al (12) showed that speech intelligibility in hearing-impaired children ( $\mathrm{CI}$ or hearing aid users) was significantly lower than the normal hearing peers. They reported that children with normal-hearing sensitivity exhibited better speech clarity than children with hearing impairment. Lee et al (13) also reported that the speech intelligibility and pitch perception in CI children were significantly lower than the normal-hearing $(\mathrm{NH})$ children.

This study aimed to compare the speech intelligibility and auditory performance skills of preschool children with normal hearing, cochlear implants, and hearing aids (HAs).

\section{Methods}

The protocol of the current analytic cross-sectional study was approved by the local ethics committee (Ethics code: R.AJUMS.REC.1398.629), which was in complete agreement with the ethical regulations of human studies set by the Helsinki declaration (2013).

\section{Participants}

During this study, 60 preschool children (aged 5 to 7 years) participated. All children were native speakers of Persian with no language disorders. Participants were divided into 3 groups of 20 children (Table 1):

- NH (control) Group: All children had an average pure-tone hearing threshold (across the test frequencies of 500-4000 Hz) at the better ear lower than $20 \mathrm{~dB} \mathrm{HL}$. They were recruited through a database of children who had participated in similar behavioral experiments at our research center but had never been exposed to the specific test materials of the current investigation.

- Hearing Aid Group: All children had a bilateral moderate or moderate to severe SNHL and used their intelligent devices at last for 6 months.

- Cochlear Implant Group: All children were selected from our national cochlear implant database (14). All children (aged 1.5 and 4 years) had bilateral severe to profound SNHL before surgery. They were unilaterally implanted and had more than 1 year of experience with their devices (MED-EL COMBI 40+) before the study. All children showed normal imaging of temporal bone and had attended regularly at postoperative aural rehabilitation sessions.

All children had IQ levels between 90 and 105 scores based on the Raven Intelligence test. They had no specific systemic, psychological, or neurological disorders. Those children who showed any symptoms of a neurodevelopmental dysfunction were excluded.

\section{Procedures}

1. Speech Intelligibility Rating (SIR): The SIR scale was used to show the degree of comprehensibility of the speaker's speech so that the listener can understand the speaker's message. This scale consisted of 5 hierarchical categories ranging from category 1 (prerecognizable words) to category 5 (connected speech intelligible to all listeners) (Table 1). The intelligibility rate of the speech improves with increasing category number (15).

2. Categories of Auditory Performance (CAP): The CAP test was utilized to measure the auditory perception. The CAP is an 8-point hierarchical rating scale. Its scores range from the lowest level ( 0 ) of being unaware of environmental sounds to the highest level (16) of having the ability to communicate through the telephone with a known person (Table 2).

3. Raven Colored Progressive Matrices (CPM): The CPM scale is an important instrument to assess intelligence that is frequently used in different contexts. The

Table 1. The speech intelligibility rating (SIR) criteria

\begin{tabular}{ll}
\hline Category & Criteria \\
\hline 1 & Connected speech is unintelligible. Pre-recognizable words in spoken language, primary mode of communication may be manual \\
2 & Connected speech is unintelligible. Intelligible speech is developing in single words when context and lip-reading cues are available \\
3 & Connected speech is intelligible to a listener who concentrates and lip-reads. \\
4 & Connected speech is intelligible to a listener who has little experience of a deaf person's speech \\
5 & Connected speech is intelligible to all listeners. Child is understood easily in everyday contexts. \\
\hline
\end{tabular}


Table 2. The categorical auditory performance (CAP) criteria

\begin{tabular}{ll}
\hline Category & \multicolumn{1}{c}{ Criteria } \\
\hline 0 & No awareness of environmental sounds \\
1 & Awareness of environmental sounds \\
2 & Response to speech sounds \\
3 & Identification of environmental sounds \\
4 & Discrimination of some speech sounds without lip- \\
5 & reading \\
6 & Understanding of common phrases without lip-reading \\
7 & Understanding of conversation without lip-reading \\
\hline
\end{tabular}

CPM consists of 3 groups of items A, Ab, and B (each group of 12 items). Then, the complete CPM test has a total of 36 items. Children were tested individually, with no time limit. The items are ordered by ascending level of difficulty in each set, as complexity among the set increases. Each test item has a drawing or a matrix with a part missing and 6 alternatives, but only 1 alternative correctly completes the figure. The minimum score is zero and the maximum score is 36 (17).

\section{Statistical Analysis}

All data analysis was performed by SPSS software Version 25 (SPSS Inc). Quantitative data were reported as means $\pm \mathrm{SD}$, and qualitative data were described as percentages and frequencies. A 1-way analysis of variance (ANOVA) test was used to compare CAP and SIR scores among the 3 groups. The statistical level was set at $\mathrm{p}<0.05$.

\section{Results}

The mean age of the participants is shown in Table 3 . There was no significant difference between different groups in terms of age variable (1-way ANOVA; $\mathrm{p}=0.711$ ). Also, there was no significant difference between the gender of the children between the 3 groups (chi-square test; $p=0.389$ ).

Table 4 shows the mean and SD of SIR and CAP tests in all 3 groups. Results of the ANOVA test showed that SIR values were significantly different among the studied groups $(\mathrm{p}<0.001)$. The post hoc analysis demonstrated that the mean SIR score in the NH children was significantly higher than in the HA $(p=0.002)$ and $C I(p=0.009)$ groups. However, the mean SIR differences between the HA and CI children were not statistically significant $(\mathrm{p}=0.885)$.

The ANOVA test results showed that a significant difference between the CAP scores in the 3 groups $(p=0.038)$. The post-hoc analysis indicated that the mean CAP scores in NH children was significantly higher than in the HA and CI participants. However, the mean SIR differences between the HA and CI children were not statistically significant $(\mathrm{p}=0.08)$.

Table 5 reveals the mean and SD of SIR scores for monolingual or bilingual children across different study groups. Our findings demonstrated that the mean SIR scores between monolingual and bilingual children were not statistically significant between $\mathrm{NH}, \mathrm{CI}$, and $\mathrm{HA}$ groups (independent sample t test; $\mathrm{p}>0.05$ ).

\section{Discussion}

Speech intelligibility can be defined as the degree to which the message of a speaker can be decoded by the listener. Speech intelligibility is one of the most important speech and language outcomes for children with hearing loss who often show large individual differences in both expressive and receptive speech and language skills. Speech intelligibility can be evaluated using several methods in hearing-impaired children; one of the most common methods is the SIR scale. The SIR has been developed to classify children's speech intelligibility according to 1 of the 5 hierarchical categories. Patients rating the scale need to judge which category is appropriate, giving the possibility that different examiners could utilize different scores in their assessments.

Our results revealed that the mean SIR scores in hearing-impaired children (CI or HA groups) were less than the $\mathrm{NH}$ group. This finding is consistent with that of Habib et al (18), Rezaei et al (12), Mok et al (19), and Heydari et al (20) who also reported that children with normal hearing sensitivity have better speech intelligibil-

Table 3. Clinical characteristics of study population

\begin{tabular}{|c|c|c|c|c|c|c|}
\hline \multirow[b]{2}{*}{ Group } & \multicolumn{2}{|c|}{ Gender } & \multicolumn{2}{|c|}{ Chronological age (Month) } & \multicolumn{2}{|c|}{ Age of amplification (Month) } \\
\hline & Male & Female & Mean & SD & Mean & SD \\
\hline Normal hearing & 6 & 14 & 71.77 & 5.05 & $\begin{array}{ll}-- \\
--\end{array}$ & $\begin{array}{ll}-- \\
--\end{array}$ \\
\hline Hearing aid & 10 & 10 & 71.45 & 10.56 & 28 & 9.81 \\
\hline Cochlear implant & 5 & 15 & 72.60 & 8.20 & 34 & 7.67 \\
\hline
\end{tabular}

SD: Standard Deviation

Table 4. Comparison of speech intelligibility scores (SIR) and categorical auditory performance (CAP) scores among different study groups

\begin{tabular}{|c|c|c|c|c|c|c|}
\hline \multirow[b]{2}{*}{ Variable } & \multicolumn{2}{|c|}{ Normal hearing } & \multicolumn{2}{|c|}{ Hearing aid } & \multicolumn{2}{|c|}{ Cochlear implant } \\
\hline & Mean & SD & Mean & SD & Mean & $\mathrm{SD}$ \\
\hline$\overline{\text { SIR }}$ & 4.85 & 0.36 & 4.10 & 0.78 & 4.20 & 0.76 \\
\hline CAP & 6.81 & 0.61 & 6.15 & 0.98 & 6.30 & 0.80 \\
\hline
\end{tabular}

SD: Standard Deviation

Table 5. Comparison of speech intelligibility scores (SIR) scores in monolingual and bilingual groups

\begin{tabular}{lcccccc}
\hline & \multicolumn{2}{c}{ Normal hearing } & \multicolumn{2}{c}{ Hearing aid } & Cochlear implant \\
\cline { 2 - 6 } Group & Mean & SD & Mean & SD & Mean & 4.09 \\
Bilingual & 4.72 & 0.46 & 3.90 & 0.71 & 0.94 \\
Monolingual & 5.0 & 0.00 & 4.33 & 0.86 & 4.33 \\
\hline SD: Standary
\end{tabular}


ity than those with CIs or HAs.

In normal-hearing listeners, depending on the speaker rate, speech material, and on-cycle duration, speech intelligibility exhibits a "U" shape. Many words remain intact at slow interruption rates, while multiple looks per word are available through interruptions at higher rates. Therefore, these 2 conditions lead to good speech intelligibility (21). In contrast to normal hearing listeners, silent interruptions in CI users strongly disrupt the speech intelligibility. For example, Chatterjee et al (22) used a 5-Hz interruption rate with sentences and found that cochlear implanted listeners' intelligibility of interrupted speech was lower than that of normal hearing subjects. As the hearing input rate increases, speech production performance will be improved and will receive more auditory feedback; therefore, it could be supposed that hearing loss can have an adverse effect on speech intelligibility.

In everyday listening situations, when the target speech is masked by competing speech or background noise, $\mathrm{NH}$ listeners are able to use top-down mechanisms to perceptually restore the degraded speech information. This phenomenon, "glimpsing," implies that the listeners could detect and integrate the glimpses of unmasked target speech parts into a speech stream using the spectrotemporal cues. Therefore, speech intelligibility in acoustical challenging situations depends on the listeners' ability to integrate the speech sounds from successive glimpses (21, $23,24)$.

Noisy environments cause a challenging condition to hearing-impaired patients in speech perception. It has been shown that spectral and temporal resolution may be affected in patients with hearing loss. Thus, in noisy situations, decreased intelligibility of intact parts and damaged bottom-up cues may negatively influence the detection and integration of the glimpses of unmasked target speech parts into a speech stream. This could lead to an obvious problem in using the top-down mechanisms in restoring and understanding degraded speech $(21,25)$.

Similar to SIR analysis, our results also indicated that the mean CAP scores in normal hearing subjects were significantly higher than the $\mathrm{CI}$ and HA children. It seems that reduced spectrotemporal cues may degrade the auditory performances in hearing-impaired children.

The current study demonstrated that SIR and CAP scores in children who used hearing aids or cochlear implants showed no significant differences. However, these values in hearing-impaired children were not very different compared with $\mathrm{NH}$ listeners' scores. It seems that children who experienced hearing loss before the development of language (ie, prelingual hearing impairment), if fitted with an appropriate hearing aid or CI early in childhood, show noticeable improvement in acquiring spoken language, particularly if they are exposed to complex language environments and supported by their parents and caregivers. However, hearing aid or CI amplifications in later childhood leads to successively less benefit, and cochlear implantation in the elementary school age or later does not lead to appropriate speech intelligibility or auditory performance skills $(26,27)$.

\section{Conclusion}

Our results revealed that speech intelligibility and auditory performance abilities in $\mathrm{NH}$ children were significantly greater than the mean scores of those with CI or HA. Furthermore, the mean scores of speech intelligibility and auditory performance abilities of the CI recipients did not significantly differ from children with HA. These findings confirm that speech intelligibility and auditory perception are 2 multidimensional phenomena that need a special rehabilitation program to further develop speaking skills.

\section{Acknowledgment}

This study was the result of a residential thesis registered in Ahvaz Jundishapur University of Medical Sciences (AJUMS), Ahvaz, Iran.

\section{Conflict of Interests}

The authors declare that they have no competing interests.

\section{References}

1. Freeman V. Speech Intelligibility and Personality Peer-ratings of Young Adults with Cochlear Implants. J Deaf Stud Deaf Educ. 2018:41-49.

2. Ashori M, Jalil-Abkenar SS. The effectiveness of cognitive rehabilitation program on auditory perception and verbal intelligibility of deaf children. Am J Otolaryngol. 2019;40(5):724-28.

3. Bazon, AC, Mantello EB, Goncales AS, Isaac ML, Hyppolito MA, Reis AC. Auditory speech perception tests in relation to the coding strategy in cochlear implant. Int Arch Otorhinolaryngol. 2016;20(3):254-60

4. Blamey P, Sarant J, Paatsch L. Relationships among speech perception and language measures in hard-of-hearing children. In: Advances in the Spoken Language Development of Deaf and HardOf-Hearing Children, P. E. Spencer.. M. Mar Shark. Oxford University Press Inc, New York. 2006: p. 85-102.

5. Ashori M. Speech intelligibility and auditory perception of pre-school children with Hearing Aid, cochlear implant and Typical Hearing. J Otol. 2020;15(2):62-66.

6. Ellis GM, Zahorik P. A dissociation between speech understanding and perceived reverberation. Hear Res. 2019;379:52-58.

7. Flanagan S, Zorila, TC, Stylianou Y, Moore BCJ. Speech processing to improve the perception of speech in background noise for children with auditory processing disorder and typically developing peers. Trend Hear. 2018;22:2331216518756533.

8. Firestone GM, McGuire $\mathrm{K}$, Liang $\mathrm{C}$, Zhang N, Blankenship CM, Xiang J, et al. A Preliminary study of the effects of attentive music listening on cochlear implant users' speech perception, quality of life, and behavioral and objective measures of frequency change detection. Front Hum Neurosci. 2020;14:110.

9. Sarafraz M, Kiani M, Bayat A, Nikakhlagh S, Karimi M, Riahi M, et al. Cortical auditory plasticity in children with cochlear implants. Electron J Gen Med. 2018:1-4.

10. Sarafraz M, Heidari M, Bayat A, Hanafi MG, Fahimi A, Farasat M, et al. Role of HRCT imaging in predicting the visibility of Round window (RW) on patients underwent cochlear implant surgery. Clin Epidemiol Glob Health. 2020;8:432-36.

11. Most T, Peled M. Perception of suprasegmental features of speech by children with cochlear implant and children with hearing aids. J Deaf Stud Deaf Educ. 2007;12(3):350-61.

12. Rezaei M, Emadi M, Zamani P, Farahani F, Lotfi G. Speech intelligibility in Persian hearing impaired children with cochlear implants and hearing aids. J Audiol IOtol. 2017;21(1):57.

13. Lee KY, van Hasselt CA, Chiu SN, Cheung DM. Cantonese tone perception ability of cochlear implant children in comparison with normal-hearing children. Int J Pediatr Otorhinolaryngol. 2002;63:13747

14. Saki N, Bayat A, Nikakhlagh S, Karimi M, Nikafrooz M, Daneshi A, 
et al. A national Iranian cochlear implant registry (ICIR): cochlear implanted recipient observational study. Int Tinnitus J. 2019;23(1):3741.

15. Allen MC, Nikolopoulos TP, O’Donoghue GM. Speech intelligibility in children after cochlear implantation. Am J Otol. 1998;19:742-46.

16. Archbold S, Lutman ME, Marshall DH. Categories of auditory performance. Ann Otol Rhinol Laryngol. 1995;166:312-14.

17. Mekik CS, Sun R, Dai DY. Similarity-based reasoning, Raven's Matrices, and general intelligence. IJCAI. 2018;18:1576-82.

18. Habib MG, Waltzman SB, Tajudeen B, Svirsky MA. Speech production intelligibility of early implanted pediatric cochlear implant users. Int J Pediatr Otorhinolaryngol. 2010;74:855-59.

19. Mok M, Holt CM, Lee K, Dowell RC, Vogel AP. Cantonese tone perception for children who use a hearing aid and a cochlear implant in opposite ears. Ear Hear. 2017;38(6):e359-68

20. Heydari S, Torabi Nezhad F, Agha Rasouli Z, Hoseyni F. Development of speech intelligibility measurement test for 3 to 5 years old normal children. Bimon Audit Tehran Univ Med Sci. 2011;20:47-53.

21. Bhargava $\mathrm{P}$, Gaudrain E, Başkent D. The intelligibility of interrupted speech: cochlear implant users and normal hearing listeners. J Assoc Res Otolaryngol. 2016;17:475-91.

22. Chatterjee M, Peredo F, Nelson D, Başkent D. Recognition of interrupted sentences under conditions of spectral degradation. J Acoust Soc Am. 2010;127:EL37-41.

23. Baskent D. Phonemic restoration in sensorineural hearing loss does not depend on baseline speech perception scores. J Acoust Soc Am. 2010;128:EL169-74.

24. Baskent D, Chatterjee M. Recognition of temporally interrupted and spectrally degraded sentences with additional unprocessed lowfrequency speech. Hear Res. 2010;270:127-33.

25. Fu QJ, Shannon RV. Effect of stimulation rate on phoneme recognition by Nucleus-22 cochlear implant listeners. J Acoust Soc Am. 2000;107:589-97.

26. Svirsky MA. Development of language and speech perception in congenitally, profoundly deaf children as a function of age at cochlear implantation. Audiol Neurootol. 2004;9:224-33.

27. Schramm D, Fitzpatrick E, Séguin C. Cochlear implantation for adolescents and adults with prelinguistic deafness. Otol Neurotol. 2002;23:698-703. 